\title{
OPEN Laser printed two-dimensional transition metal dichalcogenides
}

\author{
Omar Adnan Abbas ${ }^{1}$, Adam Henry Lewis ${ }^{1}$, Nikolaos Aspiotis ${ }^{1}$, Chung-Che Huang ${ }^{1}$, \\ loannis Zeimpekis ${ }^{1}$, Daniel W. Hewak ${ }^{1}$, Pier Sazio ${ }^{1}$ \& Sakellaris Mailis ${ }^{1,2}$
}

Laser processing is a highly versatile technique for the post-synthesis treatment and modification of transition metal dichalcogenides (TMDCs). However, to date, TMDCs synthesis typically relies on large area CVD growth and lithographic post-processing for nanodevice fabrication, thus relying heavily on complex, capital intensive, vacuum-based processing environments and fabrication tools. This inflexibility necessarily restricts the development of facile, fast, very low-cost synthesis protocols. Here we show that direct, spatially selective synthesis of 2D-TMDCs devices that exhibit excellent electrical, Raman and photoluminescence properties can be realized using laser printing under ambient conditions with minimal lithographic or thermal overheads. Our simple, elegant process can be scaled via conventional laser printing approaches including spatial light modulation and digital light engines to enable mass production protocols such as roll-to-roll processing.

Since the discovery of the indirect-to-direct bandgap transition in monolayer $\mathrm{MoS}_{2}{ }^{1,2}$, semiconducting twodimensional transition metal dichalcogenides (2D-TMDCs) have attracted significant interest due to the plethora of interesting physical properties and their compositional tunability ${ }^{3}$. Properties such as bandgap tuning via thickness reduction ${ }^{1,2}$, high mobility and on-off ratio of 2D-TMDCs based FETs ${ }^{4}$ and mechanical flexibility ${ }^{5}$ offer a broad spectrum of prospective applications in optoelectronics ${ }^{6}$, photonics $^{6}$, sensing ${ }^{7}$, nanoelectronics ${ }^{8}$, flexible and wearable electronics ${ }^{9,10}$.

Hence, finding suitable materials synthesis methods for TMDCs is essential for the industrial utility of these materials. Initial studies of 2D-TMDCs were performed in mechanically exfoliated, single/few layer, flakes from bulk crystals ${ }^{1,2}$. Mechanical exfoliation is a top down technique suitable for prototyping of devices, which however is not scalable. Liquid-phase exfoliation of TMDCs is a scalable top-down approach technique, however, thickness uniformity of liquid exfoliated TMDCs films and device performance are questionable ${ }^{11}$.

Despite the limited progress has been achieved in liquid exfoliation of TMDCs ${ }^{12}$, bottom-up synthesis approaches seem more applicable for industrial purposes. Therefore, several routes have been proposed, among them, chemical vapour deposition (CVD) has investigated extensively as this method normally produces highquality, thickness controllable crystals ${ }^{13}$. However, there are still issues faced by CVD process such as mass production of wafer-scale single crystal films, high annealing temperature requirements and long processing times.

Another bottom up method is the solution-based synthesis of 2D-TMDCs which utilizes thermal decomposition of single source precursors films such as ammonium tetrathiomolybdate $\left(\mathrm{NH}_{4}\right)_{2} \mathrm{MoS}_{4}{ }^{14}$ and ammonium tetrathiotungstate $\left(\mathrm{NH}_{4}\right)_{2} \mathrm{WS}_{4}{ }^{15}$. Recently, roll-to-roll production of few-layer $\mathrm{MoS}_{2}$ films, tens of centimetres in length with excellent long-range uniformity and stoichiometry have been demonstrated by this technique ${ }^{16}$. This promising approach could be applicable for the mass production required by industry due to cost-effectiveness and ease of growth conditions to produce large area TMDCs films. However, like CVD, this method still requires the use of a carefully controlled TMDCs growth environment, as well as standard top-down, cleanroom based lithographic post-processing for device fabrication.

Lasers have been involved in growth of 2D-TMDCs films physically via pulsed laser deposition (PLD) ${ }^{17-20}$ and chemically through in-situ laser sulphurisation ${ }^{21}$ or selenisation ${ }^{22}$ of transition metal oxides films. Nevertheless, the 2D-TMDCs films grown by PLD generally possess poor quality while laser sulphurised/selenised films cannot be isolated from untreated oxides precursor films and need special atmosphere for deposition. Moreover, due to high localised energy they can deliver, lasers have also been used for various post-treatments of 2D-TMDCs such as thinning ${ }^{23-31}$, crystallisation ${ }^{32-36}$, chemical modification (doping) ${ }^{37-45}$ and patterning ${ }^{46,47}$.

In this work, we present a bottom-up method for the direct synthesis and patterning of $\mathrm{MoS}_{2}, \mathrm{WS}_{2}$ and their heterostructures under normal room ambient operating conditions via laser-induced decomposition of

\footnotetext{
${ }^{1}$ Optoelectronics Research Centre, University of Southampton, Southampton SO17 1BJ, UK. ${ }^{2}$ Present address: Skolkovo Institute of Science and Technology, Novaya St., 100, Skolkovo 143025, Russian Federation. ${ }^{\square}$ email: s.mailis@skoltech.ru
} 


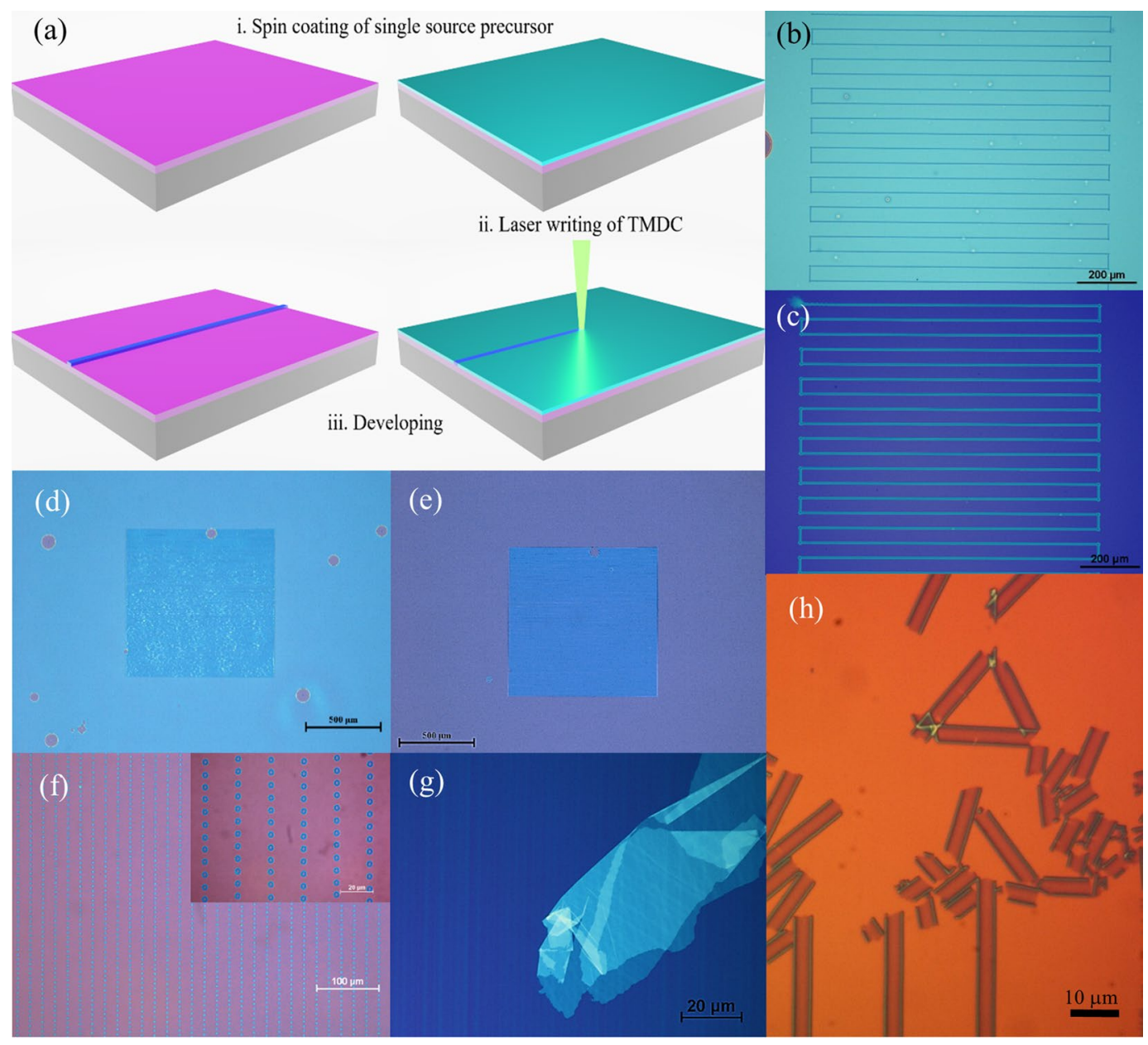

Figure 1. (a) Schematic illustration of direct laser printing of 2D-TMDCs, which comprises of: i. spin-coating of the precursor solution, ii. laser irradiation of the precursor and iii. development in organic solvent to remove unexposed precursor film. (b) Optical microscopy image of the laser irradiated $\left(\mathrm{NH}_{4}\right)_{2} \mathrm{MoS}_{4}$ precursor film. The visible serpentine tracks correspond to laser synthesized $\mathrm{MoS}_{2}$. (c) Optical microscopy image of the pattern shown in (b) after "development" step where only the precursor film is preferentially removed leaving behind the $\mathrm{MoS}_{2}$ pattern. (d,e) Optical microscopy image of laser-synthesised $\mathrm{MoS}_{2}$ film before and after development created by overlapping raster scanning. (f) Optical microscopy image of $\mathrm{WS}_{2}$ micron-size dot array (the inset corresponds to a higher magnification image of the sample). (g,h) detached $\mathrm{MoS}_{2}$ film and tracks due to excessive developing time in DMF solvent.

$\left(\mathrm{NH}_{4}\right)_{2} \mathrm{MoS}_{4}$ and $\left(\mathrm{NH}_{4}\right)_{2} \mathrm{WS}_{4}$ films, which have been applied onto various substrates. This method is also capable of creating high-precision, micro-scale 2D-TMDCs heterostructures by two-step direct laser writing.

\section{Results and discussion}

This method can be envisioned as localised laser-printing of TMDCs. The processing steps are schematically illustrated in Fig. 1a, namely: substrate conditioning and precursor solution preparation; deposition of the precursor film by spin-coating; laser exposure to locally decompose the precursor and directly synthesise and write the TMDCs patterns and finally removal of excess unexposed precursor film in a single developing step using common organic solvents. These steps are described in detail in the "Methods" section.

The optical absorption of the single source precursors was determined from the UV-Visible transmission spectra, which were obtained from various films with different thickness/molar solution concentrations, deposited by spin coating on optically transparent silica glass substrates (see Fig. S1). This analysis revealed that the precursor films exhibit considerable absorption in the green/blue region of the visible spectrum which increases proportionally with the precursor film thickness, i.e. the concentration of the precursor solution. The optimum range for the precursor solution concentrations in terms of optical absorption and film homogeneity was identified experimentally (see Fig. S1). Taking advantage of the high absorption of the precursor in the visible spectral region, it is possible to provide the energy that is required for local thermal decomposition by irradiating the film with a focussed beam of an Argon ion laser operating in a multi-wavelength mode with dominant emission at the 488 and $514.5 \mathrm{~nm}$ lines. 
In order to maximize the optical contrast of the laser synthesized TMDCs structures, $\mathrm{SiO}_{2} / \mathrm{Si}$ wafers with 285-300 nm thick oxide were the best choice of substrate for this purpose ${ }^{48}$. Figure $1 \mathrm{~b}-\mathrm{f}$ shows patterned $\mathrm{MoS}_{2}$ and $\mathrm{WS}_{2}$ that was laser-synthesised directly on $\mathrm{SiO}_{2} / \mathrm{Si}$ under ambient room temperature operating conditions. However, successful laser synthesis of $\mathrm{MoS}_{2}$ and $\mathrm{WS}_{2}$ were also performed on different substrates such as bulk silica glass and single crystal lithium niobate, as confirmed by Raman spectra (see Fig. S2), indicating that the method is substrate agnostic.

The serpentine tracks seen in Fig. 1b,c were produced by using a combination of two high precision Aerotech ABL1500 air bearing linear stages. Individual $\mathrm{MoS}_{2}$ linear tracks, or even continuous large area films can be formed using this set-up by single linear scanning or by raster scanning/overlapping of individual tracks respectively as shown in Fig. 1d,e. The laser irradiated tracks are visible under an optical microscope as shown in Fig. 1b,d as a significant optical contrast develops between the laser irradiated regions and the untreated precursor, even prior to the removal of excess precursor using DMF (dimethylformamide) solvent (see Fig. 1c,e). Similar behaviour was observed with $\mathrm{WS}_{2}$ and its precursor after developing in NMP (N-Methyl-2-pyrrolidone) solvent as shown in Fig. S3. An array of micron size $\mathrm{WS}_{2}$ islands was produced in this manner (Fig. 1f) by modulating the laser beam, using a mechanical chopper, while rastering the translation stage that supported the substrate. In the developing step, prolonged exposure of the TMDCs tracks to the DMF solvent leads to detachment and fracturing of the structures as can be seen in Fig. 1g,h due to the ease of dispersing TMDCs layers in this solvent ${ }^{11}$; however, using NMP as the developer solvent subsequently overcame this issue. However, Raman analysis of the laser-synthesised $\mathrm{MoS}_{2}$ tracks were evaluated before and after developing, suggesting that the composition of $\mathrm{MoS}_{2}$ was not altered by the development process, which involves an organic solvent (DMF) used as a developer as shown in Fig. S4.

Another notable feature arises from the Gaussian intensity profile of the laser beam, which produces a nonuniform temperature distribution across the laser irradiated area of the precursor films. As a result, the lasersynthesised TMDCs tracks exhibit a characteristic formation of side lobes located at the edges of the track that corresponds to wings of the laser intensity profile. This can be seen in the micrograph of Fig. 1h, where the darker edges of the side lobes are separated by $\sim 4 \mathrm{~m}$, matching the laser spot size. Surface profilometry measurements of these structures confirms that their shape and height is strongly influenced by both the laser processing parameters and concentration of the precursor solution, as shown in Fig. S5a. Raman spectroscopy shows that they mainly consist of thick, poor crystallinity TMDCs "hills" (Fig. S5b) in contrast to the thinner, higher crystallinity $\mathrm{MoS}_{2}$ or $\mathrm{WS}_{2}$ central regions in-between (Fig. 1h). The ratio of the area occupied by the central regions over the edges can be tuned by changing the laser intensity, writing speed and thickness of precursor film (i.e. by changing the precursor solution concentration).

It is also important to note that exploring the parameter space of direct laser synthesis will allow thickness control of the $\mathrm{MoS}_{2}$ or $\mathrm{WS}_{2}$ via sublimation of the topmost layers ${ }^{23,26}$; improve the crystallinity of the TMDCs ${ }^{32,33}$; and affect the amount of the oxide content in the structures ${ }^{37,41,42}$.

Fig. S1 shows uniform coverage and adequate optical density of the TMDCs precursor films; this required $\left(\mathrm{NH}_{4}\right)_{2} \mathrm{MoS}_{4}$ concentrations of $24 \mathrm{mM}$ and $48 \mathrm{mM}$ whereas 50, 100, 200 and $400 \mathrm{mM}$ concentrations were used for $\left(\mathrm{NH}_{4}\right)_{2} \mathrm{WS}_{4}$ spin-coating. Initially, $\mathrm{MoS}_{2}$ on $\mathrm{SiO}_{2} / \mathrm{Si}$ samples prepared with $24 \mathrm{mM}$ and $48 \mathrm{mM}\left(\mathrm{NH}_{4}\right)_{2} \mathrm{MoS}_{4}$ films were processed by irradiating multiple linear tracks using 300 and $400 \mathrm{~mW}$ power levels with a beam spot size of $3 \mu \mathrm{m}$, which was obtained with a 10×, $0.25 \mathrm{NA}$ microscope objective. Two different stage scanning speeds, 100 and $1000 \mathrm{~mm} / \mathrm{min}$ were used in these experiments. Raman spectroscopy was used to verify the formation and layer thickness of $\mathrm{MoS}_{2}$ from the characteristic peaks (in-plane $\mathrm{E}_{2} \mathrm{~g}$ ) and (out-of-plane $\mathrm{A}_{1} \mathrm{~g}$ ) and their spectral separation $^{49,50}$, with the full width half maximum (FWHM) of the peaks also providing qualitative information about the crystallinity of the laser written tracks ${ }^{14,16,51}$. Raman spectra corresponding to $\mathrm{MoS}_{2}$ films are shown in Fig. 2a. Bilayer formation was observed using the $24 \mathrm{mM}$ precursor solution at $400 \mathrm{~mW}$ laser power and $100 \mathrm{~mm} /$ min scanning speed. From Fig. 2c, which shows the spectral separation of the two characteristic Raman peaks for $\mathrm{MoS}_{2}$, it can be deduced that $\mathrm{MoS}_{2}$ films synthesised using a scanning speed of $100 \mathrm{~mm} / \mathrm{min}$ are thinner as compared to the ones synthesised with $1000 \mathrm{~mm} / \mathrm{min}$ for the same laser power. An overview of the Raman results, however, indicates that the number of $\mathrm{MoS}_{2}$ layers depends on both laser intensity and laser dwell time, which is controlled by the speed of scanning. Further Raman analysis, shown in Fig. S6c, confirms that the spectral separation between the well-pronounced in-plane $\left(E_{2} g\right)$ and out-of-plane $\left(A_{1} g\right)$ peaks is smaller for the slower writing speed, indicating thinner $\mathrm{MoS}_{2}$ layer. Notably, extended Raman spectra (up to $900 \mathrm{~cm}^{-1}$ ) on the same $\mathrm{MoS}_{2}$ tracks, shown in Fig. S6b, did not indicate formation of molybdenum trioxide (molybdenum trioxide peaks are located at $666 \mathrm{~cm}^{-1}$ and $\left.820 \mathrm{~cm}^{-1}\right)^{52}$ irrespective of synthesis in oxygen-rich, ambient conditions.

The impact of the laser writing speed on the thickness of the resulting $\mathrm{MoS}_{2}$ can be explained as follow; the local temperature of the irradiated precursor at the focal point is regulated by the laser intensity. This intensity can be controlled by the laser power and beam spot size at focus (Intensity $\mathrm{I}=\mathrm{P} / \mathrm{A}$ where $\mathrm{P}$ is the power and $\mathrm{A}$ is the irradiated area $)^{53}$. The laser induced temperature change is much faster compared with practical laser scanning speeds and so the precursor temperature dynamics reach steady state before the beam has the chance to move significantly. Therefore, the scanning speed controls the dwell time of the beam, in other words the duration of steady state heating. Consequently, the laser energy fluence ( $\times \mathrm{t}$, where $\mathrm{t}$ is the dwell time of the laser beam) delivered to the TMDCs precursors films will be higher for the films written by slower writing speed (longer interaction time) compared to faster speed (shorter interaction time) ${ }^{53}$. Longer irradiation times can result in the thinning of the synthesised $\mathrm{MoS}_{2}$ as have been demonstrated in the literature ${ }^{23}$.

Consistent formation of $\mathrm{WS}_{2}$ on $\mathrm{SiO}_{2} / \mathrm{Si}$ substrates (using 50,100, 200 and $400 \mathrm{mM}$ concentrations of $\left(\mathrm{NH}_{4}\right)_{2} \mathrm{WS}_{4}$ to create precursor films) was obtained for $300 \mathrm{~mW}$ incident laser power and a laser spot size of $1.2 \mu \mathrm{m}$ which was formed by a $20 \times, 0.4 \mathrm{NA}$ microscope objective, at two writing speeds; 100 and $1000 \mathrm{~mm} /$ min. The $\mathrm{WS}_{2}$ precursor films has lower absorption for visible light in comparison to the $\mathrm{MoS}_{2}$ precursor films (Fig S1). This means for the same irradiation conditions the local temperature of the $\mathrm{WS}_{2}$ precursor film will be 

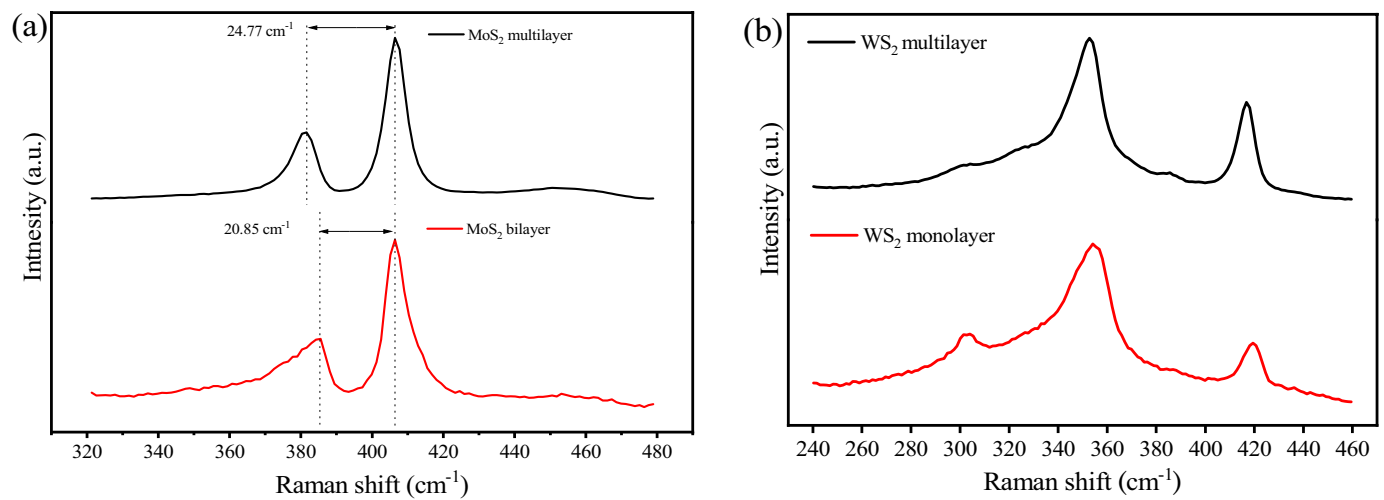

(c)

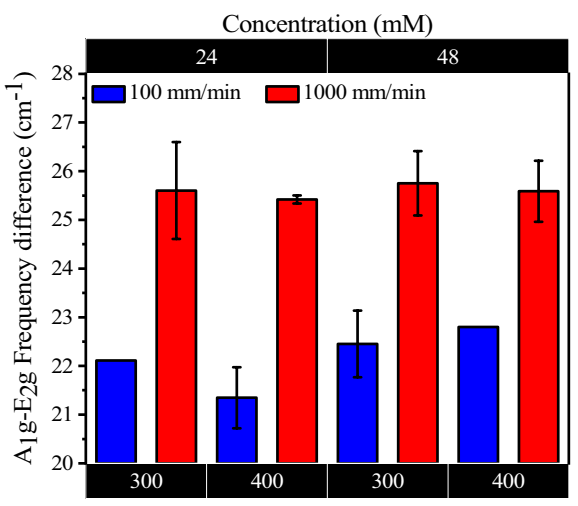

(d)

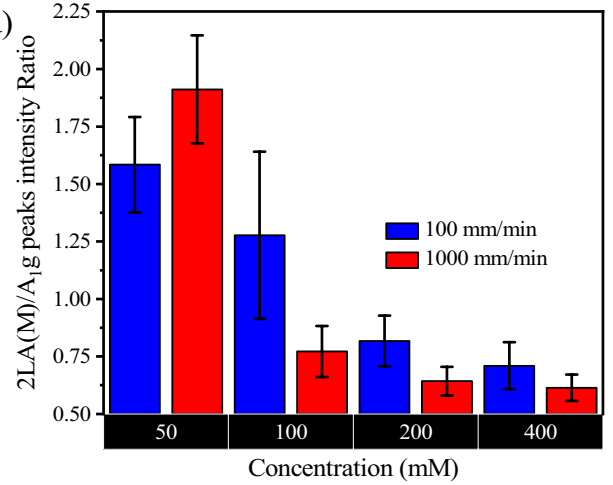

Figure 2. (a) Raman spectra corresponding to a laser synthesised bilayer (red curve) and multilayer (black curve) of $\mathrm{MoS}_{2}$. (b) Raman spectra of single layer (red curve) and multi-layer (black curve) of $\mathrm{WS}_{2}$. (c) Histogram showing the average Raman spectral separation $\left(\mathrm{A}_{1} \mathrm{~g}-\mathrm{E}_{2} \mathrm{~g}\right)$ of $\mathrm{MoS}_{2}$ as function of different laser writing conditions $(10 \times$ objective used for focusing the laser beam). The error bar represents the standard deviation for four individual $\mathrm{MoS}_{2}$ tracks. (d) Graph represents the average of Raman peak intensity ratio 2LA (M) $/ \mathrm{A}_{1} \mathrm{~g}$ of $\mathrm{WS}_{2}$ post-deconvolution as function of different laser writing conditions. The laser power was fixed to $300 \mathrm{~mW}$ and $20 \times$ objective were used to focus the laser beam. The error bars represent the standard deviation from Raman spectra obtained on five individual $\mathrm{WS}_{2}$ tracks.

lower as compared to the $\mathrm{MoS}_{2}$ precursor films. For this reason, tighter focusing using a $20 \times$ objective lens was utilised to increase the laser intensity at the irradiated spot.

As shown in Fig. S7a,b, the out-of-plane Raman mode $\mathrm{A}_{1} \mathrm{~g}$ of $\mathrm{WS}_{2}\left(\right.$ around $420 \mathrm{~cm}^{-1}$ ) was pronounced for all growth conditions. The Raman peak at around $350 \mathrm{~cm}^{-1}$ is a convolved peak that includes in-plane Raman mode $\mathrm{E}_{2} \mathrm{~g}$ and longitudinal acoustic mode $2 \mathrm{LA}(\mathrm{M})$, which arises due to the resonance with excitation wavelength ${ }^{54}$. The contribution of the 2LA (M) peak was obtained by deconvolution of the composite peak, as shown in Fig. S7c.

Figure S7c shows an example of laser written $\mathrm{WS}_{2}$ Raman spectrum where the intensity of 2LA(M) Raman peak is lower than $\mathrm{E}_{2 \mathrm{~g}}^{1}$ peak intensity. This peak ratio agrees with our previous report in few-layer $\mathrm{WS}_{2}$ films grown by thermal decomposition of the same precursor at $500{ }^{\circ} \mathrm{C}^{15}$. However, this ratio is reversed $\left(\mathrm{E}_{2 \mathrm{~g}}^{1}\right.$ peak intensity becomes lower than $2 \mathrm{LA}(\mathrm{M})$ ) when the $\mathrm{WS}_{2}$ films are annealed to a higher temperature $\left(1000^{\circ} \mathrm{C}\right)^{15}$. Moreover, the peak position of $2 \mathrm{LA}(\mathrm{M})$ is red shifted to $345 \mathrm{~cm}^{-1}$ compared to previous reports ${ }^{15,54}$ by 4-5 $\mathrm{cm}^{-1}$. Similar red shift of the $2 \mathrm{LA}(\mathrm{M})$ peak position has been previously observed in vertically oriented $\mathrm{WS}_{2}$ nanosheets ${ }^{55}$. The red shift in our laser synthesised material could be an indication of strain.

For $\mathrm{WS}_{2}$, the $2 \mathrm{LA}(\mathrm{M}) / \mathrm{A}_{1}$ g peak intensity ratio provides information about the number of layers in the film where a higher ratio corresponds to a fewer number of layers ${ }^{54}$. Figure $2 \mathrm{~b}$ shows $\mathrm{WS}_{2}$ Raman spectra corresponding to single layer and multilayer laser-synthesised films. For $\mathrm{WS}_{2}$ formed using a precursor solution concentration of $50 \mathrm{mM}$, which is the lowest concentration used in our experiments, the $2 \mathrm{LA}(\mathrm{M}) / \mathrm{A}_{1} \mathrm{~g}$ peak intensity ratio becomes 1.9 , which correspond to single layer ${ }^{54} \mathrm{WS}_{2}$. Figure $2 \mathrm{~d}$ shows a histogram of the $2 \mathrm{LA}$ $\mathrm{M}) / \mathrm{A}_{1} \mathrm{~g}$ peak intensity ratio as a function of growth conditions. The gradual decrease in peak intensity ratio with increasing precursor film concentration means that the thickness of laser-written $\mathrm{WS}_{2}$ increases proportionally. However, no significant changes in spectral separation of the $\mathrm{WS}_{2}$ peaks corresponding to layer number changes were observed (Fig. S7d), possibly due to the low sensitivity of tungsten atoms to vibrational modes changes ${ }^{56}$.

Importantly, similar to the case of laser-synthesised $\mathrm{MoS}_{2}$, despite the oxygen-rich ambient conditions associated with our direct laser synthesis and patterning method, there was no indication of significant tungsten oxide formation. As shown in Fig. S7b, a weak and broad peak appears near $700 \mathrm{~cm}^{-1}$, whereas the Raman characteristics peaks of $\mathrm{WO}_{3}$ are usually located at $710 \mathrm{~cm}^{-1}$ and $810 \mathrm{~cm}^{-157,58}$. 
The full width half maximum (FWHM) of Raman peaks can provide an indication of the crystallinity of the synthesised $\mathrm{MoS}_{2}$ and $\mathrm{WS}_{2}$ films ${ }^{59}$. The literature suggests that the crystallinity of the deposited film is affected by the annealing temperature therefore it is expected that the crystallinity of our laser synthesised films will be affected by the laser irradiation conditions. Although we did not have the means for accurate measurement of the local temperature at the irradiated spot, it is possible to assign a synthesis temperature by comparing the FWHM values that we observed to the ones found in the literature ${ }^{14,16,51,60} \cdot \mathrm{MoS}_{2}$ films grown by thermal decomposition at $500{ }^{\circ} \mathrm{C}$ exhibited FWHM values of 10 and $9 \mathrm{~cm}^{-1}$ for $\mathrm{E}_{2 \mathrm{~g}}^{1}$ and $\mathrm{A}_{1 \mathrm{~g}}$ peaks respectively ${ }^{14}$. Further annealing at $1000{ }^{\circ} \mathrm{C}$ reduced the FWHM to $6 \mathrm{~cm}^{-1}$ for both peaks ${ }^{14}$. In our laser synthesised $\mathrm{MoS}_{2}$, the minimum FWHM was around 6.75 and $5.75 \mathrm{~cm}^{-1}$. This indicates that the synthesis temperature for laser synthesised $\mathrm{MoS}_{2}$ was close to $1000^{\circ} \mathrm{C}$.

We have provided statistical analysis for FWHM of Raman peaks for both $\mathrm{MoS}_{2}$ (Fig S6d) and WS 2 (Fig $\mathrm{S} 7 \mathrm{e}, \mathrm{f})$ synthesised at different irradiation conditions, which can be used to estimate the annealing temperature for each condition. The FWHM of $\mathrm{A}_{1} \mathrm{~g}$ for $\mathrm{WS}_{2}$ was seen to decrease as a function of the precursor concentration indicating the crystallinity is improved for thicker starting precursor films (Fig. S7e).

Photoluminescence (PL) spectroscopy was also employed for probing the number of layers of laser written TMDCs films s,2,61,62. The same proprietary Raman spectroscopy system was used to obtain room-temperature PL spectra from our $\mathrm{WS}_{2}$ films, which are shown in Fig. S8. The spectra exhibit a clear concentration-dependent blue shift of the PL peak as the concentration of the precursor films spun-coated on the substrate is reduced. The PL peak appears at $1.987 \mathrm{eV}$ (624 nm wavelength) for the highest precursor concentration of $400 \mathrm{mM}$ and shifts to $2 \mathrm{eV}$ ( $620 \mathrm{~nm}$ wavelength) for $50 \mathrm{mM}$ using the same laser irradiation conditions. However, no PL was observed in $\mathrm{MoS}_{2}$ films for all laser writing conditions and precursor concentrations, possibly due to low quantum yield of $\mathrm{MoS}_{2}$ compared to $\mathrm{WS}_{2}{ }^{61}$.

Surface (stylus) profilometry was also performed on $\mathrm{MoS}_{2}$ and $\mathrm{WS}_{2}$ tracks to assess the film thickness. The profilometry measurements, which are summarized in the histograms shown in Fig. S9, are in good agreement with the Raman and PL spectroscopy analysis.

We note that numerous reports in the literature amply demonstrate that real-time, in-situ Raman spectroscopy is a highly versatile, general purpose diagnostic tool for 2D materials which can be used to analyse for example, the dynamics of laser thinning of $\mathrm{MoS}_{2}$ flakes as well as laser crystallization kinetics for amorphous $\mathrm{MoS}_{2}$ films deposited by sputtering ${ }^{29,33}$. Serendipitously, we have also discovered that micro-probe analysis (Renishaw InVia Raman Microscope operated at $532 \mathrm{~nm}$ with a $50 \times 0.75 \mathrm{NA}$ microscope objective) of our spin-coated singlesource precursor films resulted in the simultaneous laser synthesis and Raman characterisation of TMDCs layers.

Power levels of 3,22 and $40 \mathrm{~mW}$ were investigated with static exposure on $48 \mathrm{mM}$ precursor concentration films. At low power, no $\mathrm{MoS}_{2}$ or $\mathrm{WS}_{2}$ peaks were observed. Significantly, at medium power, characteristic in-plane $\left(380.3 \mathrm{~cm}^{-1}, \mathrm{E}_{2} \mathrm{~g}\right)$ and out-of-plane $\left(402.5 \mathrm{~cm}^{-1}, \mathrm{~A}_{1} \mathrm{~g}\right)$ peaks became pronounced, revealing rapid formation of $\mathrm{MoS}_{2}$. At the higher end of available pump laser power in our Raman spectroscopy setup, the same Raman peaks of $\mathrm{MoS}_{2}\left(380.3 \mathrm{~cm}^{-1}, 402.5 \mathrm{~cm}^{-1}\right)$ and $\mathrm{WS}_{2}$ (convoluted peak at $351.6 \mathrm{~cm}^{-1}$ and out-of-plane peak $415.6 \mathrm{~cm}^{-1}$ ) emerged using both single-source precursors films, as can be seen in Fig. S10.

Despite this successful demonstration, it should be noted that the performance parameters of the proprietary Raman laser system are necessarily limited by design, especially in terms of maximum power ( $40 \mathrm{~mW})$. As such, this particular system is not flexible enough to study the effect of laser power on thickness, crystallinity and oxide doping of the TMDCs films and therefore our customised, more powerful and versatile laser writing setup is essential to study thoroughly these parameters.

Further insight into the exact composition and stoichiometry as a function of the growth conditions of our films was provided by X-ray photoelectron spectroscopy (XPS). XPS investigation was performed on large area laser-synthesised $\mathrm{MoS}_{2}$ and $\mathrm{WS}_{2}$ using various precursor concentrations. Films with an area of $1 \mathrm{~mm}^{2}$, which is a large enough area for the system to collect accurate data for our specific analysis, were produced by overlapping raster scanning as in Fig. 1d,e. The XPS spectra, which were obtained from these films are shown in Fig. S11 and Fig. S12.

The second $\mathrm{Mo}^{(6+)}$ and third $\mathrm{Mo}^{(5+)}$ doublets, which can be observed in Fig. S11 correspond to molybdenum oxide. These oxide species were not detected by Raman spectroscopy, which indicates they are substitution for sulphur vacancies in the $\mathrm{MoS}_{2}$ lattice rather than individual molybdenum oxides layers ${ }^{34}$. The second doublet $\mathrm{W}^{(6+)} \mathrm{f}_{7 / 2}$ and $\mathrm{W}^{(6+)} \mathrm{f}_{5 / 2}$ in $\mathrm{W}$ core level of Fig. S12 corresponds to $\mathrm{WO}_{3}$ formation and the binding energies for this doublet is identical to that reported previously for $\mathrm{WO}_{3}{ }^{63,64}$. However, Raman spectroscopy (Fig. S7b) again did not reveal any obvious vibrational modes for the oxide. This indicates that, just as with the $\mathrm{MoS}_{2}$ layers, the films contain oxidised $\mathrm{WS}_{2}$ rather than two individual layers of $\mathrm{WS}_{2}$ and $\mathrm{WO}_{3}$ (but with a higher level of oxidation for $\mathrm{WS}_{2}$ compared to $\left.\mathrm{MoS}_{2}\right)^{34}$. We hypothesize that the oxide content in both $\mathrm{MoS}_{2}$ and $\mathrm{WS}_{2}$ films has been significantly increased compared to individual tracks because the large area, overlapping raster scanned films which are required for the XPS analysis subjected the material to prolonged laser exposure which can be considered as the equivalent of prolonged thermal annealing in an oxidizing atmosphere.

The collective results of XPS analysis, PL spectroscopy and stylus profilometry indicate that precursors films, which are prepared at lower concentrations, result in thinner TMDCs films with lower oxide content. However, the Raman spectroscopy of these thinner films showed broader Raman resonances, suggesting compromised crystallinity.

Finally, potential electronic device applications of our laser-synthesized TMDCs were explored using field effect transistor (FET) arrangements. We used two configurations: back gate, with the conductive silicon substrate as the gate electrode and an ionic top gate configuration for both $\mathrm{MoS}_{2}$ and $\mathrm{WS}_{2}$. Back-gated devices show modest performance in terms of both mobility $\left(5.5 \times 10^{-5} \mathrm{~cm}^{2} / \mathrm{V}\right.$. $\mathrm{s}$ for $\mathrm{MoS}_{2}$ and $1.1 \times 10^{-4} \mathrm{~cm}^{2} / \mathrm{V}$. $\mathrm{s}$ for WS $\mathrm{WS}_{2}$ calculated using the equation in Fig. S13) and on-off ratio (3 for $\mathrm{MoS}_{2}$ and 1.2 for $\mathrm{WS}_{2}$ ). Figure 3a shows the transfer characteristics of back gated $\mathrm{MoS}_{2}$ and $\mathrm{WS}_{2}$ FETs with device schematic. The low mobility and on-off ratio that 
(a)

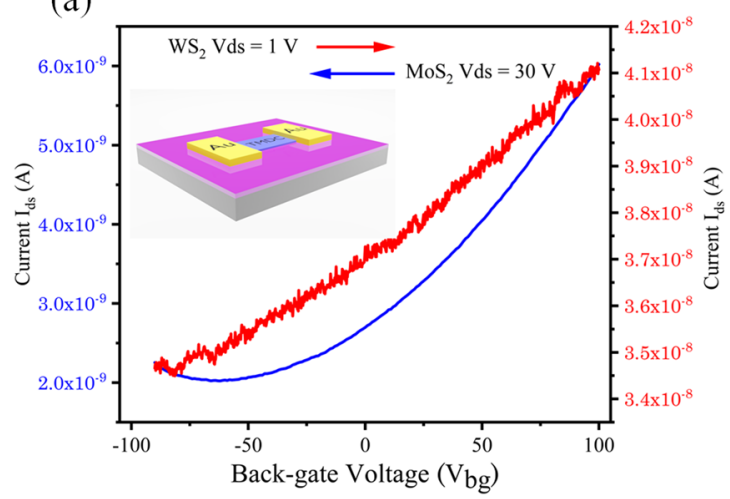

(b)

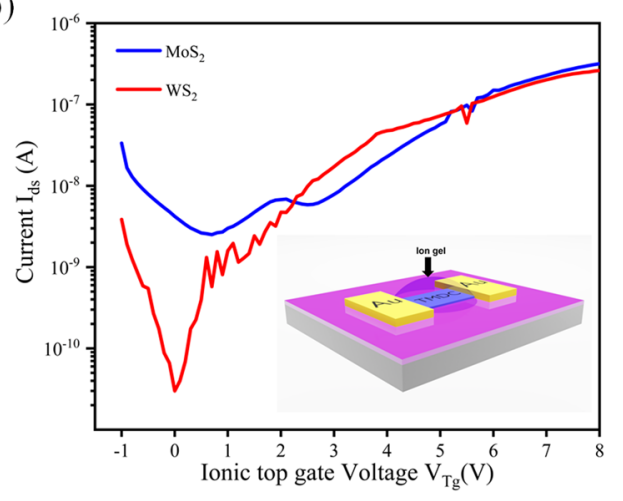

Figure 3. Transfer characteristics of laser-synthesised TMDCs-FET with (a) back-gated configuration and (b) Ionic top-gated configuration. Device schematics are also shown in inset images.

we observed are similar to other TMDCs devices reported in the literature that were also fabricated using single source precursors, but where the TMDCs channels were patterned by conventional top-down lithography $y^{14,15}$. However, when the ionic top-gate FET configuration was used, the values for both the mobility and on-off ratio improved significantly due to the higher capacitance of the ionic gate and the encapsulation of the TMDCs channels. Figure 3b shows the transfer characteristics of ionic top gated $\mathrm{MoS}_{2}$ and $\mathrm{WS}_{2}$ FETs and the device schematic. The mobility extracted for $\mathrm{MoS}_{2}$ is $7 \times 10^{-3} \mathrm{~cm}^{2} / \mathrm{V}$. s and $6 \times 10^{-3} \mathrm{~cm}^{2} / \mathrm{V}$.s for the $\mathrm{WS}_{2}$ device, while the on-off ratio for the $\mathrm{MoS}_{2} \mathrm{FET}$ is $>10^{2}$ and $\sim 10^{4}$ for $\mathrm{WS}_{2}$ which present orders of magnitude improvement over the back gated configuration.

Conventional vertical heterostructure semiconductor growth technologies have enabled revolutionary device designs, from modulation doped high mobility electron transistors, through to quantum cascade lasers. Given the plethora of available compositions and combinations, it is strongly anticipated that similar technological progress can be realised with TMDCs once a large-scale fabrication technique can be developed that allows for the facile assembly of $2 \mathrm{D}$ layers, whilst preserving sharp interfaces. Our direct laser writing approach offers the opportunity for heterostructuring of TMDCs by sequential laser synthesis of individual precursor layers to create single and multiple-overlapped tracks and arrays of $\mathrm{WS}_{2} / \mathrm{MoS}_{2}$ interfaces. Strong interlayer excitonic transitions in such type-II heterostructure systems can generate intrinsic $\mathrm{p}-\mathrm{n}$ junctions $\mathrm{s}^{65}$, ubiquitous in incumbent microelectronic devices.

Optical and scanning electron microscopy images of various $\mathrm{WS}_{2} / \mathrm{MoS}_{2}$ heterostructures are presented in Fig. 4. The array shown in Fig. 4a is formed by synthesis of a set of WS $S_{2}$ tracks separated by $20 \mu \mathrm{m}$ followed by synthesis of a set of $\mathrm{MoS}_{2}$ tracks, rotated by 90 degrees with respect to the $\mathrm{WS}_{2}$ tracks, to form a cross-hatch pattern. The unused precursor was removed in intermediate processing steps by development in NMP solvent. The heterostructures are formed at the overlapping points between the two orthogonal sets of tracks. Figure $4 \mathrm{~b}, \mathrm{c}$ shows detail of a single $\mathrm{MoS}_{2} / \mathrm{WS}_{2}$ heterostructure using optical microscopy and SEM at the same resolution. Although numerous junctions were created successfully, as can be seen in Fig. 4a and Fig. S14, some are damaged due to the non-optimised synthesis parameters. Raster scanned, overlapping $\mathrm{MoS}_{2} / \mathrm{WS}_{2}$ heterostructure are also shown as optical and SEM micrographs in Fig. 4d,f and at higher optical resolution in Fig. S14, where the individual lines forming the large area junction create a " $\operatorname{tartan"}$ pattern.

To verify the continuity of the elemental content in the patterned heterostructure we gained access to the micro-XRD beam line (I18) at Diamond Light source in Harwell campus Oxfordshire. The beamline uses a focussed X-Ray beam, with tunable energy, that was raster-scanned on the sample while simultaneously acquiring fluorescence spectra to create X-Ray fluorescence (XRF) maps of the $\mathrm{MoS}_{2} / \mathrm{WS}_{2}$ heterostructure. Figure 4e, shows such an XRF map where the Mo-Ka and W-La emission as registered in false colour (red for Mo-Ka and blue for $\mathrm{W}-\mathrm{L} \alpha$ ). The X-Ray emission of each element is spatially distinct in the areas outside the overlapping region and mixed on the heterostructure as expected. Additional evidence of the material state on the heterostructure was provided by Raman analysis of this area, as shown in Fig. 4g, where the characteristic modes of both materials are present in the heterostructure region. A large, dense (and potentially addressable) array of $50 \times 50 \mathrm{MoS}_{2} / \mathrm{WS}_{2}$ tracks and (approx. 2500) heterostructures was also imaged in false colour using the XRF facility as shown in Fig. S15. The elemental spatial distribution is again well defined, despite the close packing of the lines (50 lines $/ \mathrm{mm})$.

\section{Conclusion}

We have demonstrated a bottom-up, spatially selective laser synthesis approach that utilizes solution-based single source precursors to create patterned $\mathrm{MoS}_{2}, \mathrm{WS}_{2}$ films and their heterostructures. This method can form microscale structures with thickness down to monolayer for $\mathrm{WS}_{2}$ and bilayers for $\mathrm{MoS}_{2}$. X-ray photoelectron spectroscopy results suggest that instant decomposition of ammonium tetrathiomolybdate $\left(\mathrm{NH}_{4}\right)_{2} \mathrm{MoS}_{4}$ and ammonium tetrathiotungstate $\left(\mathrm{NH}_{4}\right)_{2} \mathrm{WS}_{4}$ can be achieved to create $\mathrm{MoS}_{2}$ and $\mathrm{WS}_{2}$ respectively even under ambient, strongly oxidizing conditions when the precursor films are exposed to localised high energy of visible 

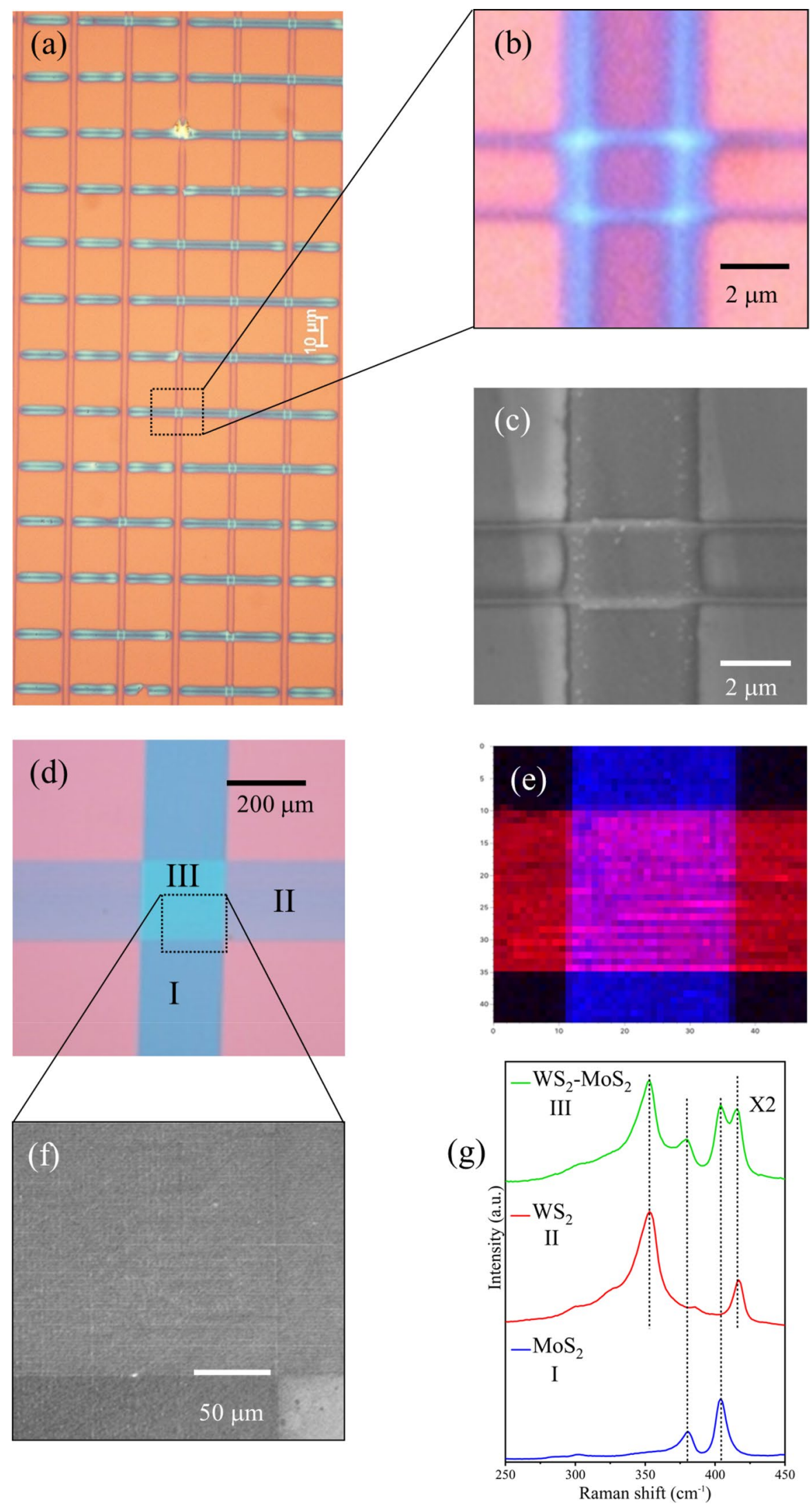

Figure 4. (a) Optical microscopy image of a $\mathrm{WS}_{2} / \mathrm{MoS}_{2}$ heterostructure array. (b,c) Optical microscopy image and Scanning Electron microscopy (SEM) of a single $\mathrm{WS}_{2} / \mathrm{MoS}_{2}$ heterostructure. (d) Optical microscopy image of an array of multiple overlapping $\mathrm{WS}_{2} / \mathrm{MoS}_{2}$ tracks forming a large area heterostructure. (e) False colour X-Ray fluorescence map of the large area $\mathrm{WS}_{2} / \mathrm{MoS}_{2}$ heterostructure shown in (d), where the red and blue bands correspond to X-Ray fluorescence from Mo and W atoms respectively. (f) Scanning Electron microscopy (SEM) of (d) showing crosshatch pattern. (g) Raman analysis of (d) is strongly indicative of a sharp vertical interface with the characteristic modes of both materials $\left(\mathrm{I}=\mathrm{MoS}_{2}, \mathrm{II}=\mathrm{WS}_{2}\right)$ present in region III. 
CW lasers. Thus, unlike TMDCs films grown by global pyrolysis of these precursors, the controlled growth environments such as low pressures and inert gases are eliminated which makes this production methodology much simpler. Additionally, due to localised decomposition which enables low-temperature processing, TMDCs structures have been successfully deposited on different types of substrates. Raman and PL spectroscopy revealed that thickness, crystallinity and oxide content of 2D-TMDCs can be tuned solely via precursor film thickness and laser writing parameters (intensity and writing speed). Field effect transistors (FETs) have been fabricated and characterised to show the potential electronic applications of 2D-TMDCs laser written structures. High-precision $\mathrm{MoS}_{2} / \mathrm{WS}_{2}$ heterostructures with lateral dimensions reaching the diffraction limit of the laser have been realised using this method by sequential laser synthesis of $\mathrm{MoS}_{2}$ and $\mathrm{WS}_{2}$ precursors.

In prospective, our laser printing process for the formation of 2D TMDCs device designs and heterostructures provides an elegant, flexible and scalable digital additive manufacturing capability. Room temperature synthesis and direct writing under ambient conditions is clearly compatible with future industrial processes in which numerous TMDCs materials could be patterned in-situ and used to augment the functionality of incumbent, bulk, wafer-based semiconductor technologies. Furthermore, fully integrated, instantaneous spectroscopic data and diagnostics including Raman, PL and pyrometry of the synthesis patterning will allow in-situ optimisation of the printed 2D materials by adjusting irradiation parameters in real time (e.g. laser fluence, duty cycles, CW or even ultrafast laser processing using nonlinear two-photon absorption for potential sub-micron patterning resolution beyond the Abbe limit). Adding these characterisation tools will not only result in a significant saving in offline analysis, but also allow for future efficient exploration of much wider parameter space than that detailed here, via the rational implementation of machine learning protocols to enable full control over the quality and number of printed TMDCs layers. Laser sublimation and thinning of layers combined with annealing and iterative processing can also improve the crystallinity as well as allowing for bandgap engineering, alloy and heterostructure formation to enable the rapid discovery of radical electronic and photonic device designs.

\section{Methods}

Substrates and precursor solution preparation. In order to ensure a high level of continuity and uniformity of laser printed TMDCs structures in terms of thickness and quality, the precursor film preparation is a critical step which must be highly reproducible. Therefore, the hydrophilicity of the substrates and the solubility of the precursors in their solutions have been optimised in the following manner:

Substrate preparation. Heavily doped n-type silicon substrates $\left(\mathrm{SiO}_{2} / \mathrm{Si}\right.$ with oxide thickness $\left.285-300 \mathrm{~nm}\right)$ were cleaved to square sizes (approx. 1 and $1.5 \mathrm{~cm}^{2}$ ). After cleaving, the substrates were cleaned sequentially by acetone, isopropanol, methanol and deionized water then dried by nitrogen gun. To promote the adhesion between the $\mathrm{MoS}_{2}$ precursor films and the substrates, Yang et al. used oxygen plasma ashing for a few seconds at low power ${ }^{51}$. However, we found this to be inadequate and instead, if the substrates are oxygen plasma treated prior to the spin coating process for 10-15 min at $0.1 \mathrm{mbar}$ pressure (oxygen flow $1000 \mathrm{~mL} / \mathrm{min}$ ) and $1000 \mathrm{~W}$ microwave power, the wettability of the $\left(\mathrm{MoS}_{2}\right.$ and $\left.\mathrm{WS}_{2}\right)$ precursor solutions will be significantly enhanced on the surface of the $\mathrm{SiO}_{2} / \mathrm{Si}$ substrates.

Preparation of precursor solutions. The initial concentration of the precursor solutions that are spin coated on the substrates play an important role on the final properties of the TMDCs structure. Therefore, the maximum concentrations have been chosen carefully to ensure optimum coverage and uniformity of precursor films after spin-coating whilst maintaining adequate optical density. In addition, this range of concentrations also ensured that unwanted clustering and film inhomogeneity were avoided. These concentrations are $24 \mathrm{mM}$ and $48 \mathrm{mM}$ for ammonium tetrathiomolybdate $\left(\mathrm{NH}_{4}\right)_{2} \mathrm{MoS}_{4}$ while the concentrations used for ammonium tetrathiotungstate $\left(\mathrm{NH}_{4}\right)_{2} \mathrm{WS}_{4}$ were 50,100, 200 and $400 \mathrm{mM}$ due to lower absorption in the visible spectral range of this precursor (see Fig. S1). The solutions of TMDCs precursors were prepared as follow:

i. $\quad \mathrm{MoS}_{2}$ precursor preparation:

Batches of $31 \mathrm{mg}$ and $62 \mathrm{mg}$ of ammonium tetrathiomolybdate $\left(\mathrm{NH}_{4}\right)_{2} \mathrm{MoS}_{4}$ were dissolved in $5 \mathrm{~mL}$ of solvent system which contains: $2 \mathrm{~mL} \mathrm{DMF}, 2 \mathrm{~mL} \mathrm{n}$-butylamine and $1 \mathrm{~mL} 2$-aminoethanol to create $24 \mathrm{mM}$ and $48 \mathrm{mM}$ concentrations of precursor solution. The solutions were sonicated for $15-20 \mathrm{~min}$ to improve the solubility and homogeneity. The solvent system was adopted with trivial modification from Yang et al. $^{51}$.

ii. $\mathrm{WS}_{2}$ precursor preparation:

Batches of 105, 209, 418 and $835 \mathrm{mg}$ of ammonium tetrathiotungstate $\left(\mathrm{NH}_{4}\right)_{2} \mathrm{WS}_{4}$ were dissolved in $6 \mathrm{~mL}$ of solvent system which contains: $3 \mathrm{~mL} \mathrm{NMP,} 2 \mathrm{~mL} \mathrm{n}$-butylamine and $1 \mathrm{~mL} 2$-aminoethanol to create 50,100, 200 and $400 \mathrm{mM}$ concentrations of precursor solution. Solutions were sonicated for $1 \mathrm{~h}$ at $70{ }^{\circ} \mathrm{C}$ to improve the solubility and homogeneity. The solvent system was adopted from our previous work, Abbas et al. ${ }^{15}$.

Spin coating. The solutions were spin coated using each precursor for $1 \mathrm{~min}$ with the following details: Step 1 -ramp $5 \mathrm{~s}$, dwell time $5 \mathrm{~s}$, rpm 500; Step 2-ramp $5 \mathrm{~s}$, dwell time $45 \mathrm{~s}, 3000 \mathrm{rpm}$ for $\mathrm{MoS}_{2}$ precursor, 6000 for $\mathrm{WS}_{2}$ precursor. After spinning, the samples were prebaked for 1 to $5 \mathrm{~min}$ to evaporate the solvents at $90^{\circ} \mathrm{C}$ for $\mathrm{MoS}_{2}$ precursor and $140^{\circ} \mathrm{C}$ for $\mathrm{WS}_{2}$ precursor. 
Laser writing parameters. The laser radiation source was a C.W. Ar ${ }^{+}$laser (Coherent Innova 90C) operating in multi wavelength mode (predominantly $\lambda=514.5 \mathrm{~nm}$ ). Laser powers in the range of 300 to $400 \mathrm{~mW}$ were used for the synthesis of $\mathrm{MoS}_{2}$ typically using a 10×, $0.25 \mathrm{NA}$ objective (laser intensities in the range of $3.1 \pm 0.1 \mathrm{MW} / \mathrm{cm}^{2}$ to $6.4 \pm 0.1 \mathrm{MW} / \mathrm{cm}^{2}$ ), whereas $\mathrm{WS}_{2}$ was synthesised using $300 \mathrm{~mW}$ of laser power and $20 \times, 0.4$ NA microscope objective (laser intensity $7.5 \pm 0.1 \mathrm{MW} / \mathrm{cm}^{2}$ ). The laser scanning speed that resulted in successful synthesis of linear tracks was in the range $100-1000 \mathrm{~mm} / \mathrm{min}$.

Developing the samples in solvents after laser writing. After laser exposure, the samples were immersed in solvents, using DMF for $\mathrm{MoS}_{2}$ or NMP for either $\mathrm{MoS}_{2}$ or $\mathrm{WS}_{2}$ for $60 \mathrm{~s}$ with gentle agitation to make sure that the untreated precursor films are totally removed (requiring multiple immersions in the solvent especially with high concentrations). After developing, the samples were dried by nitrogen gun. It is generally preferable to use NMP rather DMF for developing as the latter could cause partial lift-off of the deposited TMDCs film, as can be seen in Fig. 1g,h.

Characterisation. The transmission spectra of the precursor films were obtained using a Jasco 570 UV/Vis spectrophotometer. Raman and photoluminescence spectroscopy were performed using a Invia Raman Microscope (Renishaw) system with a $532 \mathrm{~nm}$ excitation wavelength at $20 \mathrm{~mW}$ power (unless otherwise stated) and $50 \times$ objective. Stylus profilometry was performed using KLA Tencor P-16 Stylus Profiler. XPS spectroscopy was obtained using a Thermo Fisher Scientific Thetaprobe system. SEM images of the TMDCs heterostructures were collected using a Thermo Scientific Quattro ESEM. XRF spectroscopy mapping was obtained using the microXRD beam line I18 at the Diamond Light Source, Harwell campus, Oxfordshire, UK. Electrical measurements were performed in ambient conditions using an Agilent 4155C semiconductor parameter analyser connected to a cascade micropositioning stage.

Device fabrication. Back-gated devices. The $\mathrm{MoS}_{2}$ and $\mathrm{WS}_{2}$ FET devices were directly laser printed onto $\mathrm{SiO}_{2} / \mathrm{Si}$ chips without the need for transfer from other substrates. The width of the channels $\left(200 \mu \mathrm{m}\right.$ for $\mathrm{MoS}_{2}$ and $250 \mu \mathrm{m}$ for $\mathrm{WS}_{2}$ ) were defined by overlapping raster scanning with the following parameters: solution concentration of precursor film: 48 for $\mathrm{MoS}_{2}$ and $100 \mathrm{mM}$ for $\mathrm{WS}_{2}$; Power (intensity): $300 \mathrm{~mW}$ (10×objective) for $\mathrm{MoS}_{2}$ and $250 \mathrm{~mW}(20 \times$ objective $)$ for $\mathrm{WS}_{2}$; writing speed $100 \mathrm{~mm} / \mathrm{min}$ for both; spacing $2 \mu \mathrm{m}$. After laser writing, the $\mathrm{MoS}_{2}$ film was developed in DMF and $\mathrm{WS}_{2}$ was developed in NMP to remove the untreated areas of the precursor film and form the FET channel. Source-drain ohmic contacts were fabricated using standard photolithographic protocols for metal lift-off. Subsequent e-beam evaporation was then employed with $10 \mathrm{~nm}$ $\mathrm{In} / 100 \mathrm{~nm} \mathrm{Au}$ and channel length $100 \mu \mathrm{m}$ for $\mathrm{MoS}_{2}$ FET and $10 \mu \mathrm{m}$ for WS 2 device. For photolithography, S1805 were spun coated over the samples for $1 \mathrm{~min}$ at $5000 \mathrm{rpm}$ and soft baked for $1 \mathrm{~min}$ at $110^{\circ} \mathrm{C}$. After UV exposure, the samples were developed in MF-319 developer for 5-6 s followed by rinsing in de-ionized water. It is worth mentioning that S1805 was used to avoid longer developing time in MF-319 which start damaging the $\mathrm{MoS}_{2}$ films when the developing time exceeds 10-20 s.

Ionic gel top-gated devices. The $\mathrm{MoS}_{2}$ and $\mathrm{WS}_{2}$ FET devices were directly laser printed onto $\mathrm{SiO}_{2} / \mathrm{Si}$ chips without the need for transfer from other substrates. The width of the channels ( $250 \mu \mathrm{m}$ for both) was defined by raster scanning with following parameters: solution concentration of precursor film: $48 \mathrm{mM}$ for $\mathrm{MoS}_{2}$ and $100 \mathrm{mM}$ for $\mathrm{WS}_{2}$; Power (intensity): $300 \mathrm{~mW}(10 \times$ objective $)$ for $\mathrm{MoS}_{2}$ and $250 \mathrm{~mW}(20 \times$ objective $)$ for $\mathrm{WS}_{2}$; writing speed $100 \mathrm{~mm} / \mathrm{min}$ for both; spacing $2 \mu \mathrm{m}$. After laser writing, the $\mathrm{MoS}_{2}$ film was developed in DMF and WS $\mathrm{S}_{2}$ was developed in NMP to remove the untreated areas of the precursor film and form the FET channel. Source-drain ohmic contacts were fabricated using standard photolithographic protocols for metal lift-off. Subsequent e-beam

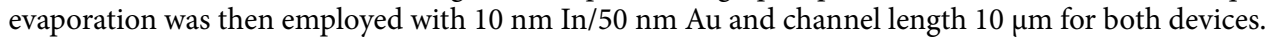

The ionic-gel gate was prepared by mixing lithium perchlorate $\left(\mathrm{LiClO}_{4}\right)$ and polyethylene oxide (PEO) with mass ratio ( $1 \mathrm{~g}$ to $0.12 \mathrm{~g}$ ) and dissolving them in $40 \mathrm{~mL}$ of methanol. The solution was heated at $50{ }^{\circ} \mathrm{C}$ until it became viscous then drop casted over the FET devices and left for one hour to dry and solidify before the measurements were undertaken. The ionic gel recipe and preparation has been adopted with trivial modifications from Hu et al. ${ }^{66}$.

Heterostructures. Single and multi-track $\mathrm{MoS}_{2} / \mathrm{WS}_{2}$ heterostructures were laser written using the following steps: the substrates were cleaned and plasma conditioned prior to spin-coating of the $\mathrm{WS}_{2}$ precursor solution with $100 \mathrm{mM}$ concentration. Subsequently, the $\mathrm{WS}_{2}$ structures were synthesised using laser power at 250 $\mathrm{mW}, 20 \times$ objective and $100 \mathrm{~mm} / \mathrm{min}$ writing speed. Next, the $\mathrm{WS}_{2}$ sample was developed in NMP to remove the untreated precursor film. Afterwards, the $\mathrm{MoS}_{2}$ precursor solution with concentration $48 \mathrm{mM}$ was spun-coated directly on the $\mathrm{WS}_{2}$ laser-processed sample and the $\mathrm{MoS}_{2}$ lines written perpendicularly to $\mathrm{WS}_{2}$ tracks using laser power $200 \mathrm{~mW}, 20 \times$ objective and $100 \mathrm{~mm} / \mathrm{min}$ writing speed. Finally, the sample was immersed again in NMP to develop the $\mathrm{MoS}_{2} / \mathrm{WS}_{2}$ heterostructures. The reason behind this sequence of precursor film deposition is that laser formation of $\mathrm{WS}_{2}$ essentially requires higher laser intensity which might be damaging to $\mathrm{MoS}_{2}$ structures if they were written first.

The observed damage to the $\mathrm{WS}_{2}$ tracks in Fig. $4 \mathrm{a}$ and Fig. S14 after the second developing step when the $\mathrm{MoS}_{2}$ precursor film was cast, is most likely due to the $\mathrm{MoS}_{2}$ precursor solution containing DMF which could partially dissolve the $\mathrm{WS}_{2}$ structures. Thus, further optimisation is needed for double-layer deposition of precursor films and for the laser writing conditions to create large, dense and addressable heterostructure arrays. 


\section{Data availability}

All experimental data required to support the findings of this work are presented either in the main article or the supplementary information.

Received: 3 September 2020; Accepted: 11 January 2021

Published online: 04 March 2021

\section{References}

1. Mak, K. F., Lee, C., Hone, J., Shan, J. \& Heinz, T. F. Atomically thin $\mathrm{MoS}_{2}$ : A new direct-gap semiconductor. Phys. Rev. Lett. 105, 136805 (2010).

2. Splendiani, A. et al. Emerging photoluminescence in monolayer $\mathrm{MoS}_{2}$. Nano Lett. 10, 1271-1275 (2010).

3. Chhowalla, M. et al. The chemistry of two-dimensional layered transition metal dichalcogenide nanosheets. Nat. Chem. 5, 263-275 (2013).

4. Radisavljevic, B., Radenovic, A., Brivio, J., Giacometti, V. \& Kis, A. Single-layer $\mathrm{MoS}_{2}$ transistors. Nat. Nanotechnol. 6, 147-150 (2011).

5. Pu, J. et al. Highly flexible $\mathrm{MoS}_{2}$ thin-film transistors with ion gel dielectrics. Nano Lett. 12, 4013-4017 (2012).

6. Mak, K. F. \& Shan, J. Photonics and optoelectronics of 2D semiconductor transition metal dichalcogenides. Nat. Photonics 10, 216-226 (2016).

7. Li, B. L. et al. Low-dimensional transition metal dichalcogenide nanostructures based sensors. Adv. Funct. Mater. 26, 7034-7056 (2016).

8. Wang, Q. H., Kalantar-Zadeh, K., Kis, A., Coleman, J. N. \& Strano, M. S. Electronics and optoelectronics of two-dimensional transition metal dichalcogenides. Nat. Nanotechnol. 7, 699-712 (2012).

9. Akinwande, D., Petrone, N. \& Hone, J. Two-dimensional flexible nanoelectronics. Nat. Commun. 5, 5678 (2014).

10. Yao, J. \& Yang, G. 2D group 6 transition metal dichalcogenides toward wearable electronics and optoelectronics. J. Appl. Phys. 127, $030902(2020)$.

11. Coleman, J. N. et al. Two-dimensional nanosheets produced by liquid exfoliation of layered materials. Science 331, 568-571 (2011).

12. Lin, Z. et al. Solution-processable 2D semiconductors for high-performance large-area electronics. Nature 562, 254-258 (2018).

13. Zhang, Y. et al. Recent progress in CVD growth of $2 \mathrm{D}$ transition metal dichalcogenides and related heterostructures. Adv. Mater. 31, 1901694 (2019).

14. Liu, K.-K. et al. Growth of large-area and highly crystalline $\mathrm{MoS}_{2}$ thin layers on insulating substrates. Nano Lett. 12, 1538-1544 (2012).

15. Abbas, O. A. et al. Solution-based synthesis of few-layer $\mathrm{WS}_{2}$ large area continuous films for electronic applications. Sci. Rep. 10, $1696(2020)$.

16. Lim, Y. R. et al. Roll-to-roll production of layer-controlled molybdenum disulfide: A platform for 2D semiconductor-based industrial applications. Adv. Mater. 30, 1705270 (2018).

17. Loh, T. A. J., Chua, D. H. C. \& Wee, A. T. S. One-step synthesis of few-layer $\mathrm{WS}_{2}$ by pulsed laser deposition. Sci. Rep. 5, 18116 (2015).

18. Serna, M. I. et al. Large-area deposition of $\mathrm{MoS}_{2}$ by pulsed laser deposition with in situ thickness control. ACS Nano 10, 6054-6061 (2016).

19. Tian, K., Baskaran, K. \& Tiwari, A. Growth of two-dimensional $\mathrm{WS}_{2}$ thin films by pulsed laser deposition technique. Thin Solid Films 668, 69-73 (2018).

20. Siegel, G., Venkata Subbaiah, Y. P., Prestgard, M. C. \& Tiwari, A. Growth of centimeter-scale atomically thin $\mathrm{MoS}_{2}$ films by pulsed laser deposition. APL Mater. 3, 56103 (2015).

21. Huang, C. C. et al. Transfer-free growth of atomically thin transition metal disulfides using a solution precursor by a laser irradiation process and their application in low-power photodetectors. Nano Lett. 16, 2463-2470 (2016).

22. Chen, Y. Z. et al. Ultrafast and low temperature synthesis of highly crystalline and patternable few-layers tungsten diselenide by laser irradiation assisted selenization process. ACS Nano 9, 4346-4353 (2015).

23. Castellanos-Gomez, A. et al. Laser-thinning of $\mathrm{MoS}_{2}$ : On demand generation of a single-layer semiconductor. Nano Lett. 12, 3187-3192 (2012).

24. Lu, J. et al. Improved photoelectrical properties of $\mathrm{MoS}_{2}$ films after laser micromachining. ACS Nano 8, 6334-6343 (2014).

25. Hu, L., Shan, X., Wu, Y., Zhao, J. \& Lu, X. Laser thinning and patterning of $M_{2} S_{2}$ with layer-by-layer precision. Sci. Rep. 7, 15538 (2017).

26. Bissett, M. A., Hattle, A. G., Marsden, A. J., Kinloch, I. A. \& Dryfe, R. A. W. Enhanced photoluminescence of solution-exfoliated transition metal dichalcogenides by laser etching. ACS Omega 2, 738-745 (2017).

27. Venkatakrishnan, A. et al. Microsteganography on $\mathrm{WS}_{2}$ monolayers tailored by direct laser painting. ACS Nano 11, 713-720 (2017).

28. Park, J., Kim, M. S., Cha, E., Kim, J. \& Choi, W. Synthesis of uniform single layer $\mathrm{WS}_{2}$ for tunable photoluminescence. Sci. Rep. 7, 16121 (2017).

29. Gu, E. et al. A real-time Raman spectroscopy study of the dynamics of laser-thinning of $\mathrm{MoS}_{2}$ flakes to monolayers. AIP Adv. 7, 125329 (2017)

30. Park, J. et al. Composition-tunable synthesis of large-scale $\mathrm{Mo}_{1-\mathrm{x}} \mathrm{W}_{\mathrm{x}} \mathrm{S}_{2}$ alloys with enhanced photoluminescence. ACS Nano 12, 6301-6309 (2018).

31. Gong, L. et al. Emergence of photoluminescence on bulk $\mathrm{MoS}_{2}$ by laser thinning and gold particle decoration. Nano Res. 11, 4574-4586 (2018).

32. McConney, M. E. et al. Direct synthesis of ultra-thin large area transition metal dichalcogenides and their heterostructures on stretchable polymer surfaces. J. Mater. Res. 31, 967-974 (2016).

33. Vilá, R. A. et al. In situ crystallization kinetics of two-dimensional $\mathrm{MoS}_{2} .2 D$ Mater. 5, 11009 (2017).

34. Sirota, B., Glavin, N. \& Voevodin, A. A. Room temperature magnetron sputtering and laser annealing of ultrathin $\mathrm{MoS}_{2}$ for flexible transistors. Vacuum 160, 133-138 (2019).

35. Wuenschell, J. K. \& Helvajian, H. Enhanced laser crystallization of thin film amorphous molybdenum disulfide $\left(\mathrm{MoS}_{2}\right)$ by means of pulsed laser ultrasound. Opt. Express 27, 5859-5873 (2019).

36. Heyne, M. H., de Marneffe, J.-F., Radu, I., Neyts, E. C. \& De Gendt, S. Thermal recrystallization of short-range ordered WS ${ }_{2}$ films. J. Vac. Sci. Technol. A 36, 05G501 (2018).

37. Lu, J. et al. Atomic healing of defects in transition metal dichalcogenides. Nano Lett. 15, 3524-3532 (2015).

38. Tran Khac, B. C. et al. Laser-induced particle adsorption on atomically thin $\mathrm{MoS}_{2}$. ACS Appl. Mater. Interfaces 8, 2974-2984 (2016).

39. He, Z. et al. Revealing defect-state photoluminescence in monolayer $\mathrm{WS}_{2}$ by cryogenic laser processing. ACS Nano 10, 5847-5855 (2016).

40. Ma, S. et al. Enhanced photocatalytic activity of $\mathrm{WS}_{2}$ film by laser drilling to produce porous $\mathrm{WS}_{2} / \mathrm{WO}_{3}$ heterostructure. Sci. Rep. 7, 3125 (2017) 
41. Atkin, P. et al. Laser exposure induced alteration of $\mathrm{WS}_{2}$ monolayers in the presence of ambient moisture. 2 D Mater. 5, 15013 (2017).

42. Alrasheed, A. et al. Surface properties of laser-treated molybdenum disulfide nanosheets for optoelectronic applications. ACS Appl. Mater. Interfaces 10, 18104-18112 (2018).

43. Afaneh, T., Sahoo, P. K., Nobrega, I. A. P., Xin, Y. \& Gutiérrez, H. R. Laser-assisted chemical modification of monolayer transition metal dichalcogenides. Adv. Funct. Mater. 28, 1-7 (2018).

44. Drndić, G. D. Laser-induced fabrication of nanoporous monolayer $\mathrm{WS}_{2}$ membranes. 2 D Mater. 5, 35011 (2018).

45. Kim, E. et al. Site selective doping of ultrathin metal dichalcogenides by laser-assisted reaction. Adv. Mater. 28, 341-346 (2015).

46. Kim, S. W. et al. Patterning of periodic ripples in monolayer $M_{2}$ by using laser irradiation. J. Korean Phys. Soc. 69, 1505-1508 (2016).

47. Rani, R. et al. Controlled formation of nanostructures on $\mathrm{MoS}_{2}$ layers by focused laser irradiation. Appl. Phys. Lett. 110, 83101 (2017).

48. Li, H. et al. Rapid and reliable thickness identification of two-dimensional nanosheets using optical microscopy. ACS Nano 7, 10344-10353 (2013).

49. Lee, C. et al. Anomalous lattice vibrations of single- and few-layer $\mathrm{MoS}_{2}$. ACS Nano 4, 2695-2700 (2010).

50. Li, H. et al. From bulk to monolayer $\mathrm{MoS}_{2}$ : Evolution of Raman scattering. Adv. Funct. Mater. 22, 1385-1390 (2012).

51. Yang, J. et al. Wafer-scale synthesis of thickness-controllable $\mathrm{MoS}_{2}$ films via solution-processing using a dimethylformamide/nbutylamine/2-aminoethanol solvent system. Nanoscale 7, 9311-9319 (2015).

52. Windom, B. C., Sawyer, W. G. \& Hahn, D. W. A raman spectroscopic study of $\mathrm{MoS}_{2}$ and $\mathrm{MoO}_{3}:$ Applications to tribological systems. Tribol. Lett. 42, 301-310 (2011).

53. Hashemzadeh, M. et al. The application of specific point energy analysis to laser cutting with $1 \mu \mathrm{m}$ laser radiation. Phys. Proc. 56, 909-918 (2014).

54. Berkdemir, A. et al. Identification of individual and few layers of $\mathrm{WS}_{2}$ using Raman Spectroscopy. Sci. Rep. 3, 1755 (2013).

55. Shifa, T. A. et al. A vertical-oriented $\mathrm{WS}_{2}$ nanosheet sensitized by graphene: An advanced electrocatalyst for hydrogen evolution reaction. Nanoscale 7, 14760-14765 (2015).

56. Zeng, H. \& Cui, X. An optical spectroscopic study on two-dimensional group-VI transition metal dichalcogenides. Chem. Soc. Rev. 44, 2629-2642 (2015).

57. Gutiérrez, H. R. et al. Extraordinary room-temperature photoluminescence in triangular $\mathrm{WS}_{2}$ monolayers. Nano Lett. 13, 34473454 (2013).

58. Chen, T. Y. et al. Comparative study on $\mathrm{MoS}_{2}$ and $\mathrm{WS}_{2}$ for electrocatalytic water splitting. Int. J. Hydrog. Energy 38, 12302-12309 (2013).

59. McDevitt, N. T., Zabinski, J. S., Donley, M. S. \& Bultman, J. E. Disorder-induced low-frequency Raman band observed in deposited $\mathrm{MoS}_{2}$ films. Appl. Spectrosc. 48, 733-736 (1994).

60. McDevitt, N. T., Zabinski, J. S. \& Donley, M. S. The use of Raman scattering to study disorder in pulsed laser deposited $\mathrm{MoS}_{2}$ films. Thin Solid Films 240, 76-81 (1994).

61. Zhao, W. et al. Evolution of electronic structure in atomically thin sheets of $\mathrm{WS}_{2}$ and $\mathrm{WSe}_{2}$. ACS Nano 7, 791-797 (2013).

62. Zeng, H. et al. Optical signature of symmetry variations and spin-valley coupling in atomically thin tungsten dichalcogenides. Sci. Rep. 3, 1608 (2013).

63. Sun, Y. et al. Photoelectrochemical and structural characterization of carbon-doped $\mathrm{WO}_{3}$ films prepared via spray pyrolysis. Int. J. Hydrog. Energy 34, 8476-8484 (2009).

64. Benoit, A., Paramasivam, I., Nah, Y. C., Roy, P. \& Schmuki, P. Decoration of $\mathrm{TiO}_{2}$ nanotube layers with $\mathrm{WO}_{3}$ nanocrystals for high-electrochromic activity. Electrochem. Commun. 11, 728-732 (2009).

65. Kang, K. et al. Layer-by-layer assembly of two-dimensional materials into wafer-scale heterostructures. Nature 550, 229-233 (2017).

66. Hu, H. et al. Broadly tunable graphene plasmons using an ion-gel top gate with low control voltage. Nanoscale 7, 19493-19500 (2015).

\section{Acknowledgements}

The first author gratefully acknowledges the financial support from The Higher Committee for Education Development in Iraq (HCED Iraq). The authors acknowledge financial support from the UK's Engineering and Physical Sciences Research Council (EPSRC) through National Hub in High Value Photonic Manufacturing (grant EP/ N00762X/1). The authors would like to thank Diamond Light Source for beamtime, Dr. K. Ignatyev along with Diamond Light Source staff for their assistance on beamline I18 (proposal SP17304) that contributed to the XRF results presented in this article. We also thank Jon Gorecki for his assistance in preparation of the ionic gel.

\section{Author contributions}

O.A.A, P.S. and S.M. conceived the idea and composed the manuscript. O.A.A. designed the experiments, prepared the samples for laser writing, analysed the Raman data, fabricated and characterized the FET devices. O.A.A. and S.M. performed the laser writing experiments. A.H.L performed the Raman and Photoluminescence spectroscopy. I.Z. and N.A. performed the XPS spectroscopy and analysed its data. C.H.H. helped with preparation of precursor films. The whole work was supervised and managed by D.H., P.S. and S.M.

\section{Competing interests}

The authors declare no competing interests.

\section{Additional information}

Supplementary Information The online version contains supplementary material available at https://doi. org/10.1038/s41598-021-81829-w.

Correspondence and requests for materials should be addressed to S.M.

Reprints and permissions information is available at www.nature.com/reprints.

Publisher's note Springer Nature remains neutral with regard to jurisdictional claims in published maps and institutional affiliations. 
(c) (i) Open Access This article is licensed under a Creative Commons Attribution 4.0 International cc) License, which permits use, sharing, adaptation, distribution and reproduction in any medium or format, as long as you give appropriate credit to the original author(s) and the source, provide a link to the Creative Commons licence, and indicate if changes were made. The images or other third party material in this article are included in the article's Creative Commons licence, unless indicated otherwise in a credit line to the material. If material is not included in the article's Creative Commons licence and your intended use is not permitted by statutory regulation or exceeds the permitted use, you will need to obtain permission directly from the copyright holder. To view a copy of this licence, visit http://creativecommons.org/licenses/by/4.0/.

(C) The Author(s) 2021 